\title{
Entrainment of respiration to repetitive finger tapping
}

\author{
JOHN THOMAS WILKE, ROBERT W. LANSING, and CECIL A. ROGERS \\ University of Arizona, Tucson, Arizona 85721
}

Subjects were instructed to synchronize finger tapping with visual signals of various frequencies. It was found that breathing rate became entrained to tapping rate if the latter fell within a range of two breaths per minute of the subject's previous breathing rate. Entrainment did not occur when the subject merely monitored the visual stimuli but only during performance of tapping. The effect occurred without. subjects' awareness and was observed only in the absence, of deliberate adjustments in respiratory frequency on the part of the subject. Temporal restrictions on the neural events responsible for motor output appear to be responsible for the phenomenon.

The brainstem respiratory centers emit a periodic output to the respiratory muscles. The frequency of this output is set by metabolic and mechanical factors, so that humans at rest maintain adequate alveolar ventilation with minimal output of muscular energy (Campbell, Agostoni, \& Davis, 1970). Resting respiratory frequency can be adjusted, however, through a rather wide range with negligible change in the total work of breathing (Mead, 1960; Otis, Fenn, \& Rahn, 1950 ), on the long term by an increase in tidal volume, and for a few successive breaths by a subthreshold change in blood $\mathrm{O}_{2}$ tension. When such changes in breathing rate are nonrandom, they have functional significance in terms of other behaviors of the organism.

The behavioral control of breathing is well known in an active organism-certain behaviors alter breathing patterns for prolonged periods of time, and in some cases the tempo of movements sets the frequency of respiratory rhythms. During playing of wind instruments or speaking, the individual must adopt a breathing pattern which permits both the performance of the task and the meeting of metabolic needs (Bouhuys, 1964; Bunn \& Mead, 1971). In exercise requiring rhythmic body movements, respirations often become synchronized with the movements of other parts of the body (Asmussen, 1964; Astrand, 1970; Christensen, 1932).

In an organism at rest, however, when metabolic demand is not altered, the question of whether or not other periodic motor events might entrain breathing rate to their own frequency has not previously been addressed. In this paper, we manipulate the frequency of a motor act employing small muscles not involved in respiration and monitor the frequency and timing of respiration in an otherwise resting subject.

John Thomas Wilke's present address is: Western Ohio Branch Campus, Wright State University, Celina, Ohio 45822. Cecil A. Rogers' present address is: Department of Psychology, Augusta College, Augusta, Georgia 30904.

\section{METHODS}

Four male college-age volunteers served as subjects. Each was told that the purpose of the experiment was to determine his ability to tap at certain rhythms and that this in turn would reflect his musical and dancing aptitudes. He was also told that it would be necessary to monitor his level of relaxation and that the only equipment available was the pneumotachograph mouthpiece. A nose clip was used.

Tidal volume, air flow, and tapping were continuously recorded as the resting subject pressed a microswitch of very low mechanical resistance in time with periodic signals displayed by means of an oscilloscope. The frequency of these signals was set at a value near five times the subject's breathing rate; instructions had been given that he was to count these signals and time each tap to coincide with each fifth signal. The tap consisted of an index finger movement of approximately $5 \mathrm{~mm}$. The particulars of the cue signals and tapping task employed in the experiment were chosen after many alternatives were tried. The task decided upon best met three criteria: (1) it sufficiently occupied the subject's information processing capacity that he could not simultaneously attend to his breathing, (2) its execution did not appreciably affect respiratory drive, and (3) the experimenter could precisely control the subject's rate of tapping without interrupting it simply by varying the rate of the visual signals.

The procedure followed with each subject began with a determination of his "basal" respiratory rate. Next, by adjusting the frequency of the cue signals, tapping at the same rate as the subject's breathing was induced. Then the frequency of cue signals was changed in a step-wise fashion and effects upon breathing rate measured.

Air flow was recorded using a No.2 Fleisch pneumotachograph and a Grass PT5 pressure transducer; tidal volumes were obtained by integration of the air flow signal with a Grass 7P10 integrator. The cue signals were produced and controlled by a Grass S8 square wave stimulator. Data were recorded with a Grass Model 7 polygraph.

\section{RESULTS}

Figure 1 shows a typical entrainment epoch. Such synchrony was observed at three different rates for each of the four subjects (Table 1). The mean range of entrainment across subjects was 2.1 breaths per minute (BPM), with Subject R.C. showing the smallest range (1.7 BPM) and Subject M.E. showing the largest 
A
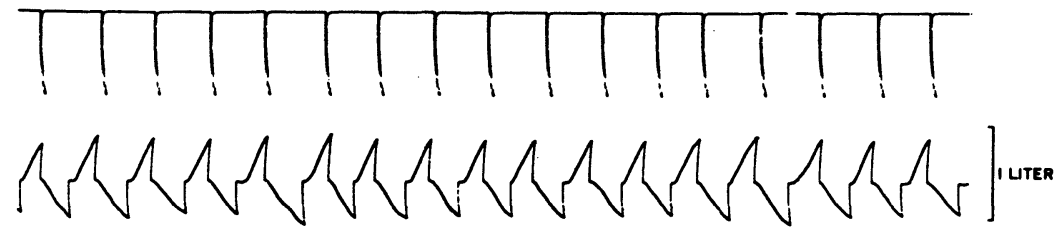

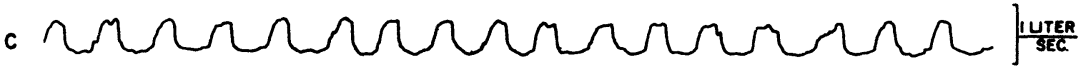

Figure 1. The phase synchronization of tapping and breathing. A. Each pen deflection represents a finger tap. B. Respiratory volume (inspiration upward). C. Air flow (inspiration upward) Subject M. E.

(2.6 BPM). The experimental increases and decreases of respiratory frequency did not fail to occur when attempted within these limits. Neither the magnitude nor the direction of such consistent changes would be predicted on the basis of random fluctuations in the breathing rate of a resting subject but were predictable from the subject's rate of tapping. The altered breathing rates could be maintained for at least 10 to 20 breaths simply by maintaining tapping at that rate. One method of demonstrating that the period of each breath was closely related to the interval between taps is to look at phase synchronization statistically. A breath-by-breath analysis of the time of occurrence of the tapping response indicated that in nearly all instances of entrainment which persisted for more than a few successive breaths, the tap occurred during the first 300 msec of the expiratory phase. A statistical study of the variances of the timing of the tapping response with respect to the respiratory cycle was carried out on all three epochs of entrainment for all four subjects. The results of tests of the heterogeneity of these variances showed that in all instances of entrainment observed in the present experiment the variance of the point in the respiratory cycle at which tapping occurred was less $(p<.05)$ than that found with the same subject at a slightly different tapping rate which was out of the range of the observed entrainment effect (see Table 2). Thus, the synchronization observed cannot be accounted for in terms of chance fluctuations in breathing or tapping.
This analysis also shows that entrainment is an all or nothing phenomenon, in the sense that if the tapping rate moves out of the range of the effect, synchrony abruptly disappears. Moreover, careful examination of the original records revealed that the synchrony was not due to adjustments in tapping rate. That the changes in breathing rate were not brought about by fluctuations in need to breathe as tapping frequency changed was shown by analysis of minute volume recordings.

Other evidence that the phenomenon observed was in fact an entrainment of breathing upon tapping, although convincing, was not amenable to statistical analysis. For example, at the limits of entrainment, respiratory adjustments occurred to keep in phase synchronization with tapping: at several points during the experiment when a fast tapping rate was induced, respiratory rate would increase and remain in synchrony for a few breaths, then skip a tap, then resume perfect synchrony until, presumably, $\mathrm{O}_{2}$ accumulation overrode the entraining mechanism. The counterpart of this at low tapping rates also occurred-an abnormally slow respiratory rate would be locked in for a few breaths, an extra breath interjected during the interval between two taps, then another epoch of synchrony. Furthermore, as illustrated in Figure 1, slight irregularities in tapping rate were accompanied by identical fluctuations in the period of each breath, again pointing to the close phase synchronization which characterizes this entrainment effect.

Table 1

Mean Frequencies of Tapping and Respiration

\begin{tabular}{|c|c|c|c|c|c|c|}
\hline & \multicolumn{3}{|c|}{ Subject R.C. } & \multicolumn{3}{|c|}{ Subject D.C. } \\
\hline & Epoch 1 & Epoch 2 & Epoch 3 & Epoch 1 & Epoch 2 & Epoch 3 \\
\hline $\begin{array}{l}\text { Cue Signals/Min } \\
\text { Breaths/Min } \\
\text { Mean Time Lag* } \\
\text { Minute Volume } \dagger\end{array}$ & $\begin{array}{c}19.1 \\
18.8 \\
135 \\
9.16\end{array}$ & $\begin{array}{c}16.9 \\
17.1 \\
112 \\
8.77\end{array}$ & $\begin{array}{c}17.8 \\
18.2 \\
194 \\
8.72\end{array}$ & $\begin{array}{c}17.3 \\
17.3 \\
97 \\
5.83\end{array}$ & $\begin{array}{c}19.2 \\
19.7 \\
155 \\
6.32\end{array}$ & $\begin{array}{c}16.9 \\
16.9 \\
1514 \\
5.46\end{array}$ \\
\hline Minute Volume $\dagger$ & \multicolumn{3}{|c|}{ Subject M.E. } & \multicolumn{3}{|c|}{ Subject J.J. } \\
\hline $\begin{array}{l}\text { Cue Signals/Min } \\
\text { Breaths/Min } \\
\text { Mean Time Lag* } \\
\text { Minute Volume }\end{array}$ & $\begin{array}{c}14.3 \\
14.3 \\
285 \\
6.11\end{array}$ & $\begin{array}{c}16.9 \\
16.9 \\
110 \\
6.71\end{array}$ & $\begin{array}{c}16.0 \\
16.1 \\
244 \\
7.13\end{array}$ & $\begin{array}{l}17.9 \\
18.1 \\
42 \\
12.31\end{array}$ & $\begin{array}{c}20.4 \\
20.4 \\
638 \\
12.65\end{array}$ & $\begin{array}{l}18.3 \\
18.8 \\
61 \\
11.66\end{array}$ \\
\hline
\end{tabular}

*Mean time lag (milliseconds) between onset of expiration and tap

†Liters/min 
For two subjects, an effort was made to determine whether breathing rate was truly synchronizing with the motor act itself, or merely with the monitored visual cue stimuli. For these subjects, additional trials were run in which instructions had been given not to tap but to continue counting the cue stimuli as before, keeping track of every fifth one. Comparison of respiratory rate adjustments under this signal counting condition with those during tapping in synchrony with the same cue stimuli was made. The difference between rate of cue signals and rate of breathing during "signal counting only" was compared with the difference between tapping rate and rate of breathing during "tapping." The analysis for each subject was carried out on three separate epochs for each of the two conditions, and each epoch within a condition exemplified a different cue signal rate. A Mann-Whitney U test found that the difference between tapping and respiratory frequencies was significantly less than that between signal counting and respiratory frequencies (U[5.5,32.0], $\mathrm{p}<.05$ ). Therefore, since synchronization did not occur while the subject was merely counting the cue stimuli, entrainment to these stimuli could not have been taking place, supporting the conclusion that the synchrony observed in the present experiment was an entrainment to the frequency of the simple motor act of tapping.

A further condition was tested on two subjects to determine whether the "entrainment" might be nothing more than the voluntary exertion of control over breathing rate on the part of the subject. That subjects had no knowledge that their breathing and tapping rates were identical was verified by questioning each subject after the session. But, more definitively, Figure 2 illustrates the gross deterioration of breathing rate which occurred when the subjects were instructed to deliberately synchronize breathing with tapping at a rate which only moments before had produced entrainment without this instruction. It was impossible for the subject to attend to the cue stimuli to maintain the required tapping rate and simultaneously exert
Table 2

F Ratios for Tests of Heterogeneity of Variances*

\begin{tabular}{ccccc}
\hline \multirow{2}{*}{$\frac{F 1}{F 2}$} & \multicolumn{4}{c}{ Subjects } \\
\cline { 2 - 5 } & R.C. & D.C. & M.E. & J.J. \\
\hline Nonentrainment Epoch & 12.119 & 99.403 & 5.745 & 79.279 \\
Entrainment Epoch 1 & $(10,9)$ & $(12,7)$ & $(10,9)$ & $(11,11)$ \\
Nonentrainment Epoch & 37.040 & 82.488 & 22.617 & 4.380 \\
Entrainment Epoch 2 & $(10,7)$ & $(12,9)$ & $(10,14)$ & $(11,11)$ \\
Nonentrainment Epoch & 9.832 & 78.520 & 13.595 & 7.679 \\
Entrainment Epoch 3 & $(10,11)$ & $(12,6)$ & $(10,16)$ & $(11,8)$ \\
\hline
\end{tabular}

*The variance of the difference in time of occurrence between each tap and the beginning of an expiration for an epoch of at least $1 \mathrm{~min}$.

deliberate control over his breathing. Only after nearly $4 \mathrm{~min}$ of continuous practice and careful instruction as to how fast to breathe, when to exhale, etc., were the subjects able to approximate the synchrony they had previously produced. Even then, simple inspection of air flow patterns clearly differentiated "voluntary" from "automatic" control of breathing.

\section{DISCUSSION}

Neurological and behavioral evidence support the view that there are central nervous system mechanisms for the nonmetabolic control of the respiratory musculature which are similar to those involved in the regulation of other forms of somatomotor activity (Plum, 1970; Sears, 1971). The present study has found that respiration is temporally integrated with other motor events, and the finding that expirations tend to coincide with the motor act agrees with results obtained by Simpson (1973). Thus, it is possible that the nometabolic control of respiratory rate is accomplished by the same neurophysiological mechanisms which impose a particular frequency upon other motor
Figure 2. Tapping, respiratory volume, and air flow for Subject $J$. J. when instructed to force his breathing rate into synchronization with his taps. A continuous record, more than 3 min long.
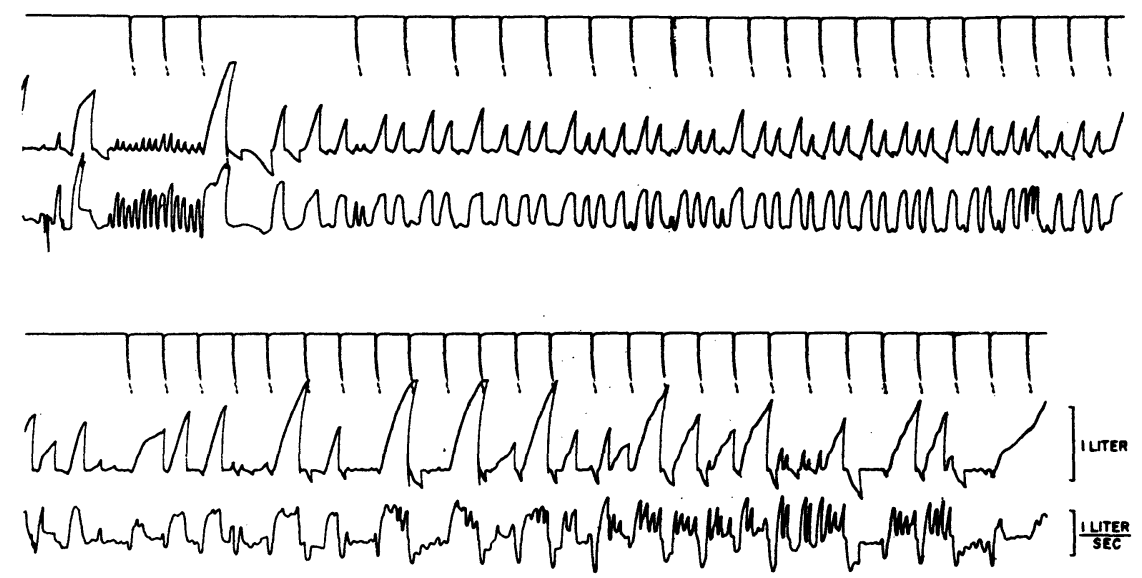
acts-for instance, the gait of a pedestrian which falls into synchrony with a passing band, periodicity in reaction time distributions (White \& Harter, 1969), intermittent sampling of input for hand control (Navas \& Stark, 1968), and the psychological moment (Murphree, 1954; White, 1963). In other words, there appears to be a temporal as well as spatial constraint upon the output of the neural control mechanism for breathing. Further, the precise time of occurrence of the relevant motor commands is neither of functional importance in terms of homeostasis nor is it random.

The link between respiration and other perceptual-motor events has already been demonstrated in another context. For example, Buschbaum and Callaway (1965) found that simple reaction time is faster during expiration than inspiration, and Flexman et al. (1974) found that visual signals presented during expiration were detected more frequently than those presented during inspiration. When these data are taken together with the results of the present finding, that taps tend to occur during expiration, three different hypotheses regarding the physiological mechanism involved can be realistically considered. First of all, Buschbaum et al, in explaining their findings, suggested that vagal discharge inhibits motor output during inspiration. While the entrainment findings of the present study do not discredit this interpretation, they cannot be accounted for by it either.

The second possibility, as pointed out by Flexman et al., argues that since the brain is relatively uninvolved in monitoring the passive expiratory phase, there would be less "neural interference" than during inspiration, thus accounting for the improved signal detectability during expiration found by these investigators. This interpretation can also explain the present data: assuming that response-produced feedback from tapping creates neural activity in the kinesthetic sensory system of the CNS, this source of information would arrive during a time when more processing capacity is available if the tap occurs early in expiration. Since the experimental arrangement does not permit adjustment of tapping frequency to accomplish this, the CNS causes inspirations, which require processing capacity, to alternate with taps, resulting in phase synchrony of expiration and tapping.

The third possibility is that the CNS possesses a highly sophisticated mechanism for producing synergy among diverse neural events which operate within the same frequency range. Such a mechanism would operate upon a principle similar to that of feedforward and would cause all changes of state to temporally coincide, thus perhaps minimizing total energy expenditure, or at least freeing the nervous system for other functions in the intervals between pulses of activity. It is significant in this regard that in all but one case of entrainment in the present experiment, the tap and the change of state from inspiration to expiration were separated by less than about $300 \mathrm{msec}$, and, in the one exceptional case, the tap coincided with the change of state from expiration to inspiration. Another consideration which appears to lend some support to the synergy notion is found in a study by Smith (1973), who found that subjects' voluntary respiratory tracking is superior during tracking of a visual display of their own hand movements as compared to tracking of a display not produced by their own motor acts. Although Smith's study employed voluntary tracking and the results reported in the present study were found with "involuntary" entrainment, the findings suggest a timing mechanism which integrates various forms of motor output in both situations.

Available data on the temporal organization of neurophysiological activity cries out for an adequate construct. The three hypotheses given here are simply suggestions, and the hope is that readers who find them unacceptable will begin to assemble relevant experimental findings in support of better ones. The time seems to be right for added emphasis upon the orchestration of diverse simultaneous nervous system functions, which although subtle and difficult to demonstrate empirically, is after all the superordinate function of the nervous system for the individual organism interacting with its environment. Perhaps in losing our awe of the nervous system as neurophysiology has advanced, we have forgotten that the system as a whole is more sophisticated than the sum of our concepts of the capabilities of its individual components. There is no doubt that the nervous system operates on some principle which maximizes precise and efficient integration of function. The present authors view the present study as one small example of the operation of that principle.

\section{REFERENCES}

Asmussen, E. "Muscular exercise." In W. O. Fenn and H. Rahn (Eds.), Handbook of Physiology (Sec. 3, Respiration, Vol. 2). Baltimore, Md: Williams \& Wilkins, 1964. Pp. 939978.

Astrand, P. O., \& Rodahl, K. Textbook of Work Physiology. New York: McGraw-Hill, 1970.

BounuYs, A. Lung volumes and breathing patterns in windinstrument players. Jourral of Applied Physiology, 1964, 19. 967-975.

BunN, J. C., \& MEAD, J. Control of ventilation during speech. Journal of Applied Physiology, 1971, 31, 870-872.

Buschbaum, M., \& Callaway, E. Influences of respiratory cycle on simple reaction time. Perceptual \& Motor Skills, $1965,20,961-966$.

Campbell, E. J. M., Agostoni, E., \& Davis, J. N. The respiratory muscles. Philadelphia and London: Saunders, 1970.

Christensen, E. H. Beitrage zur Physiologie schwerer korperlicher Arbeit. VI. Der Stoffwechsel und die respiratorischen Funktionen bei schwerer korplicher arbeit. Arbeitephysiologie, 1932, 5, 463-478.

Flexman, J. E., Demaree, R. G., \& Simpson, D. D. Respiratory phase and visual signal detection. Perception \& Psychophysics, 1974, 16, 337-339. 
MEAD, J. Control of respiratory frequency. Journal of Applied Physiology, 1960, 15, 325-336.

MurPhreE, O. D. Maximum rates of form perception and the alpha rhythm: An investigation and test of current nerve net theory. Journal of Experimental Psychology, 1954, 48, 57-61.

Navas, F., \& Stark, L. Sampling or intermittency in hand control system dynamics. Biophysics Journal, 1968, 8, 252-302.

OTIS, A. B., FENN, W. O., \& RAHN, H. Mechanics of breathing in man. Journal of Applied Physiology, 1950, 2, 592-607.

PLUm, F. "Neurological integration of behavioral and metabolic control of breathing." In R. Porter (Ed.), Breathing: Hering-Brener Centenary Symposium. London: Churchill, 1970. Pp. 151-175.

SEARs, T. A. "Breathing: A sensori-motor act." In I. Gilliland \& J. Francis (Eds.), The scientific basis of medicine annual reviews. London: Athlone Press, 1971. Pp. 129-147.
Simpson, D. D. Patterns of respiratory behavior. Physiological Psychology, 1973, 1, 141-146.

Smith, K. U. "Feedback of the motor system." In J. D. Maser (Ed.), Efferent organization and the integration of behavior. New York: Academic Press, 1973.

White, C. T. Temporal numerosity and the psychological unit of duration. Psychological Monograph, 1963, 77, (12, Whole No. 575).

WhITE, C. T., \& HARTER, M. R. Intermittency in reaction time and perception and evoked response correlates of image quality. Acta Psychologica, 1969, 30, 368-377.

(Received for publication May 8, 1975.) 